\title{
Systematical Analysis of the Application of Chinese Traditional Medicine Informatics to Diabetes Proved Recipesw
}

Jie YANG

Correspondence: yangjie@nju.edu.cn

State Key Laboratory of Pharmaceutical Biotechnology, Life Science College, Nanjing University, Nanjing 210093, P. R. China.

\begin{abstract}
Chinese medicinal materials that originate from animal, mineral and plant have thousands years for diabetes therapy and accumulate a great deal of valuable clinical experience. The rapidly increasing diabetes mellitus is becoming a serious threat to mankind health in all parts of the world. Currently, bioinformatics has already impenetrate biology, medicine, genomics, clinical pharmacology, botany, and other subjects. This paper mainly expatiates the actual application of information technology to traditional Chinese medicinal materials, especially build a database of diabetes mellitus proved recipe (DMPR) by Microsoft Access 2000; analyze and find complexity information of DMPR viz matrix alignment. Our research results reveal that there is not linearity relationship between $P$ value and clinical activities of DMPR that show diversity and complexity; and t-test results show that $\mathrm{P} 1$ value of simple score matrix and $\mathrm{P} 2$ value of complex score matrix present significantly difference and the latter is more rational than the former because the secondary score matrix plays a complementary role to the first score matrix so that enhance the reliability and the veracity of prediction. Therefore, bioinformatics at many scales and many levels should be spread for Chinese traditional medicine proved recipe possessing complexity, diversity, and non-linearity. This will greatly change the research actuality of Chinese traditional medicine, improve the research level of Chinese traditional medicine informatics, and accelerate the research step of modernization of Chinese traditional medicine.
\end{abstract}

Keywords: diabetes mellitus proved recipe, bioinformatics, Chinese Traditional Medicine Informatics, diabetes Chinese traditional prescriptions

\section{Introduction}

Diabetes mellitus is chronic metabolic disorder in the endocrine system and systemic underway disease with the equilibrium maladjustment and metabolic turbulence of sugar, protein, fat, water, electrolyte, and vitamine, and is becoming the third "killer" of the health of mankind along with cancer, cardio- and cerebroascular diseases because of its high prevalence, morbidity and mortality [1]. Diabetes mellitus is mostly divided into two types: type 1 (insulin-dependent diabetes mellitus, IDDM) and type 2 (noninsulin-dependent diabetes mellitus, NIDDM). Type 1 , accounting for $5-10 \%$ of diabetes, in which the body does not produce any insulin due to islet beta-cell apoptosis, most often occurs in children and young adults [2]. Type 2, accounting for $90-95 \%$ of diabetes, in which the body does not produce enough, or properly use, insulin due to the damage of insulin gene, its receptor gene, glucokinase gene, and mitochondria gene, is nearing epidemic proportions, due to an increased number of elderly people, and a greater prevalence of obesity and sedentary lifestyles $[3,4]$. As a complex disease that stems from an interaction of environmental and genetic factors (i.e. obesity and lack of exercise), NIDDM is characterized by defects in insulin secretion and action including both hepatic and extrahepatic insulin resistance with increased beta-cell apoptosis. Hallmarks of the disease are insulin resistance in skeletal muscle, liver and fat, combined with relative insulin insufficiency due to a decline in beta-cell function [5]. And ethnic and racial differences have been found in heterogeneous populations within the same area. As a rule, incidence is highest in Scandinavian countries, intermediate in the US, Spain, and Israel, and lowest in Asian and most Latin American countries. Most researchers believe that, in the presence of a genetic predisposition, something in the environment triggers the development of diabetes [6,7].

Up to now, the control and treatment of diabetes and its complications (such as hypertension, brain stroke, coronary heart disease, diabetic retinopathy, chronic kidney disease, etc.) mainly depend on the chemical or biochemical agents, namely insulin, insulin-like growth factor, sulfonylureas, biguanide, alpha-glucosidase inhibitors, aldose reductase inhibitor, and others. But the fact is that it has never been reported that someone had recovered totally from diabetes. Chinese medicine performed a good complex and holistic system of medical practice with its own philosophy, diagnosis, treatment systems and pharmacology and is showing a bright future in the therapy of diabetes mellitus and its complications [8]. It considers the human body in relation to its own natural, physical and social environment and employs its own language, systems of logic, and criteria for understanding health and diagnosing illness.

(C) 2012 Jie Yang; licensee Herbert Publications Ltd. This is an open access article distributed under the terms of Creative Commons Attribution License 
The practice of Chinese medicine involves physical therapy using acupuncture, moxibustion and related disciplines such as Tuina Massage and Qi Gong and chemical therapy using Chinese medicinal materials (Chinese material medica, CMM). Chinese herbal medicine, an integral part of the traditional Chinese medicine and has played an important role in the medical care of Chinese people over the past several thousand years $[\mathbf{8}, \mathbf{9}]$. Moreover, CMM treat diabetes mainly by two aspects: integrated care of the body and controlling and lowing blood glucose to a normal level. On the one hand, the treatment is based on the principle of tonifying five viscera, benefiting jing and qi, and removing blood stasis to adjusting yin-yang balance, enriching $q i$ and blood, and reinforcing viscera, etc. Here, jing and $q i$ are the most essential matter composed of body. The former means a liquid existing in body viscera, which is transformed into the latter (a vital energy) in order to promote, regulate, and control various body functions. On the other hand, CMM strictly possess certain lowering blood glucose activity by some different approaches as follows: stimulating beta-cell of pancreatic islet to release insulin, restraining glucagons secretion, increasing the number of insulin receptor, enhancing the appetency and sensitivity of insulin receptor site to insulin (e.g. berberine and phlorizin), improving the post-effect of insulin receptor (e.g rhubarb), inhibiting gluconenogenesis (the leading-out of glycogen), promoting glucose utilization in the tissue and organ, postponing intestine absorbability of glucose, clearing away free radicals, resisting lipid peroxidation and correcting the metabolic disorder of lipid and protein (such as lycium barbarum fruit) [10], improving microcirculation in the body. More than 28 ginsenosides have been extracted from ginseng, and might be associated with a wide range of therapeutic actions in the CNS and cardiovascular and endocrine systems. Indeed, ginseng promotes immune function and metabolism, and possesses anti-stress and anti-aging activities. Several ginsenosides were proven to be non-organ-specific tumor suppressors and to improve learning and memory in patients with Alzheimer's disease. Danshensuan is isolated from dānshēn (Salvia miltiorrhiza), which limits not only the progression but also the symptoms of several cardiovascular diseases, an effect that is probably related to its antioxidant activity. Lycium barbarum fruit water decoction, its crude polysaccharide extracts, and its purified polysaccharide fractions possess substantial antioxidant activity, hypoglycemic and hypolipidemic effects [10]. Based on a large number of chemical and pharmacological research work, numerous bioactive compounds have been found in Chinese medicinal plants for diabetes, such as (English, Latin and Chinese pinyin name in order for each herb) lotus leaf Folium nelumbinis (Héhuā), Indian trumpetflower seed Semen oroxyli (Mùhúdié), senna leaf Folium sennae (Fānxièyè), shorthorned epimedium herb Herba epimedii (Yínyánghè), good days tea leaf Gynostemma pentaphyllum (Jiāogŭlán), green tea leaf Camellia sinensis (L-chá), etc [11]. Recently, some research of diabetes were developed by acupuncture, which reveal that needle pricking certain points, such as accupunctive point of spleen, accupunctive point of midriff, Zúsānlĭ point of crus, Xïnjing of heart, Fèijing of lung, etc., could enhance body sensitivity to insulin and reduce blood glucose.

Note that bioinformatics nowadays has an essential role both, in deciphering genomic, transcriptomic, and proteomic data generated by high-throughput experimental technologies, and in organizing information gathered from traditional biology and medicine. The evolution of bioinformatics is now going to be directed towards recently emerging areas of integrative and translational genomics, and ultimately personalized medicine, especially in drug target identification and validation and in the development of biomarkers and toxicogenomic and pharmacogenomic tools to maximize the therapeutic benefit of drugs [12]. Integrated computational and experimental programmes are being developed, with the goal of enabling in silico pharmacology by linking the genome, transcriptome and proteome to cellular pathophysiology [13]. Clinical proteomics is an emerging field that involves the analysis of protein expression profiles of clinical samples for de novo discovery of disease-associated biomarkers and for gaining insight into the biology of disease processes [14]. At present, bioinformatics has impenetrated biology and medicine and strides forward pharmacy and Chinese traditional medicines, which play a major role in the pursuit of future biological discoveries and medical applications [15]. This present paper addresses the key attempts to propose ways for future research and development for CMM, especially the application of Chinese medicinal materials informatics to diabetes mellitus proved recipe.

\section{Materials and Methods}

There are hundreds of prescriptions to aim directly at different symptoms of diabetes, such as Compound Recipes (prescription with more than two medicines) and Simple Recipes (prescription with one medicine), and a lot of natural medicines and preparations are used in these prescriptions or folk Simple Recipes and diets for diabetes care in China, most of which come from plants. A large number of research work on these medicines and their chemical constituents, experimental and clinical anti-diabetic activity have been conducted yet. DMPRs compiled here come from a book named by Diabetes Chinese Medicinal Proved Recipes [16], consisting of 233 Chinese traditional prescriptions (proved recipes) and containing 241 kinds of Chinese traditional medicine (traditional Chinese herb), which are commonly used in the traditional Chinese medical system and have demonstrated clinical anti-diabetic effectiveness, involving type 1 diabetes, type 2 diabetes, and its complications. Among these natural medicines, 216 originate from plants, 22 from animals or insects, and 3 from mines. It is strongly significant to pay close attention to traditional Chinese medical therapeutics and natural medicines for treatment of diabetes mellitus and 
its complications.

\section{Database of diabetes mellitus proved recipes and statistic analysis}

Database of DMPRs was compiled by Microsoft Access 2000, which collects 233 Chinese herbal prescriptions for the clinical treatment of diabetes and incorporates search function. The database is composed of serial number, name of DMPRs, sorts, traditional Chinese herb composition of the prescriptions, adaptive symptom, usage method, clinical effect, the author of and origin of the prescriptions, etc. Simultaneously, it can inquire and search based on the name of the prescriptions, name of authors, and traditional Chinese herbs. And it helps for the following statistic analysis and further matrix comparison. According to our methods $[17,18]$, the accumulative times $\left(c_{k}\right)$ and the percentage $\left(P_{k}\right)$ of each herbal medicine in 233 DMPRs (Table 1) were analyzed and calculated by symbolic statistic method as follows:

\section{Score matrix}

$$
\mathrm{P}_{k}=c_{k} \times \frac{1}{233} \times 100 \%
$$

\section{Simple score matrix method}

On the basis of our methods [18], according to the order of 241 kinds of $C M M s$, the matching direction of medicinal herbs, and the character of drug pairs (mated herbs), choose 18 kinds of CMM from eight DMPRs (Table 2) for a $1 \times 18$ unitary score matrix consisting of zero for scoring mismatches and a series of positive scores namely (7 73333333322221111) on the diagonal for scoring 18 kinds of CMM match with each other. Take the sum of times of each CMM existing in training set as a score standard, namely $M=\sum_{i=1}^{n} m_{i}=50, n=18$ Here, $m_{i}$ are the times of each herb existing in training set.

\section{Complex score matrix method}

Similarly, choose 10 kinds of CMMs from eight DMPRs (Table 2) consisting of a first score matrix $1 \times 10$ consisting of zeros for scoring mismatches and a series of positive scores namely (6 432222211 ) on the diagonal. Take the sum of times of each herb existing in training set as a score standard, namely $S=\sum_{i=1}^{n} s_{i}=25, n=10$. Here, $s_{i}$ are the times of each herb existing in training set.

Considering that some drug pairs play important roles in treating diabetes, introduce some mated herbs to first score matrix to construct a secondary score matrix. On the first score matrix, we thereby convert the score values of another corresponding herb in drug pairs (Table 3 ) as follows:

$s_{2}=s_{1} \times \frac{c_{k p}}{c_{k p^{\prime}}}$, which make up of a secondary score matrix

$1 \times 12$. Here, $s_{1}$ and $s_{2}$ express the score $\left(s_{i}\right)$ of each herb in first matrix and the relative score of each corresponding herb of drug pairs in secondary matrix (integer), respectively; a group of drug pairs was tagged by subscripts ${ }_{p}$ and ${ }_{p^{\prime}}$ and the former for the secondary matrix and the later for the first matrix. $C_{k p}$ and $C_{k p}$ figure the accumulative times of a group of drug pairs (Table 1) and then obtain a secondary score matrix $1 \times 12$ consisting of zeros for scoring mismatches and a series of positive scores namely (4 322222211111111 ) on the diagonal.

\section{Evaluation of CDMR}

Based on the simple score matrix, estimate and evaluate the others (225 DCMPs) with the exception of training set's DCMPs. According to the score rule of bioinformatics [19], take a score of some DCMP on the basis of the proved recipe's composition. If the composition of some proved recipe is the same of that in the training set, its mark in some proved recipe equals to the mark in the training set plus 1 ; if the composition of some proved recipe is different from that in the training set, its mark in some proved recipe is zero. Then the total score $(A)$ of some proved recipe is described as follows:

$$
A=\sum\left(q_{j}\right) q_{j}= \begin{cases}\sum_{j=1}^{n}\left(m_{j}+1\right) & (j \in i), \text { where } i \text { and } j \\ 0 & (j \notin i)\end{cases}
$$

denote the compositions of the training set and some proved recipe, respectively; $q_{j}$ shows the times of each herb existing in some proved recipe; $j \in i$ or $j \notin i$ express if the composition of proved recipe falls in that of training set or not. And take the percentage of the total score $(A)$ of some proved recipe to the score standard of training set for evaluation the pending recipes, which is generally figured as follows: $P_{1}=A / M \times 100 \%$. Generally speaking, $P$ values drop into the scope, viz. $0 \leq P \leq 1$.

On the other hand, similar to above-mentioned simple matrix method, based on the first score matrix and with the aid of the secondary score matrix, estimate and evaluate the other 225 DMPRs. According to the score rule of bioinformatics $[18,19]$, give a mark of some DMPR on the basis of the proved recipe's composition. When the same herbs of between the proved recipe and training set aren't more than 5, if the composition of some proved recipe is the same of that in the training set, its mark in some proved recipe equals to the mark in the training set plus 1 ; if the composition of some proved recipe is different from that in the training set, its mark in some proved recipe is zero. Then the total score $(A)$ of some proved recipe is described as follows: $A=\sum\left(q_{j}\right) q_{j}=\left\{\begin{array}{ll}\sum_{j=1}^{n}\left(s_{j}+1\right) & (j \in i) \\ 0 & (j \notin i)\end{array}\right.$. Here, menas the score of each herb existing in the first matrix and the secondary matrix. When the same herbs of between the proved recipe and training set are more than 5 , if the composition of some proved recipe is the same of that in the training set, its mark in some proved recipe equals to the mark in the training set; if the composition of some proved recipe is different from that in the training set, its mark in some proved recipe is zero. The 
Jie Yang. Journal of Diabetes Research and Clinical Metabolism 2012, http://www.hoajonline.com/journals/pdf/2050-0866-1-10.pdf

Table 1. The order of 241 kinds of key Chinese medicinal materials (CMM) in diabetes Chinese traditional prescriptions

\begin{tabular}{|c|c|c|c|c|}
\hline No. & $\begin{array}{l}\text { Herb name in Chinese } \\
\text { pinyin }\end{array}$ & English and Latin name in order for each herb (CMM) * & $c_{k}$ & $\mathrm{P}_{k}(\%)$ \\
\hline 1 & Huángqí & membranous milkvetch root Radix Astragali seu Hedysari ${ }^{0,1}$ & 153 & 65.66 \\
\hline 2 & Dānshēn & danshen root Radix salviae miltiorrhizae & 118 & 50.64 \\
\hline 3 & Shānyào & common yam rhizome Rhizoma dioscoreae ${ }^{0,2}$ & 97 & 41.63 \\
\hline 4 & Shēndihuáng & rehmannia dried rhizome Radix rehmanniae $e^{0,1}$ & 94 & 40.34 \\
\hline 5 & Dāngguī & Chinese angelica Radix Angelicae Sinensis ${ }^{0}$ & 73 & 31.33 \\
\hline 6 & Gĕgēn & lobed kudzurine root Radix puerariae ${ }^{0,2}$ & 67 & 28.76 \\
\hline 7 & Shā̄nzhūyú & asiatic cornelian cherry fruit Fructus Corni ${ }^{0,1}$ & 64 & 27.47 \\
\hline 8 & Tiānhuāfěn & mongolian snakegourd root Radix Trichosanthis ${ }^{0,2}$ & 57 & 24.46 \\
\hline 9 & Chuānxióng & szechwan lovage rhizome Rhizoma Chuanxiong & 55 & 23.61 \\
\hline 10 & Fúlíng & tuckahoe dried sclerotium Poria ${ }^{0}$ & 53 & 22.75 \\
\hline 11 & Xuánshēn & figwort root Radix Scrophulariae ${ }^{0,2}$ & 45 & 19.31 \\
\hline 12 & Gŏuqǐzĭ & lycium barbarum fruit Fructus lycii ${ }^{0,2}$ & 45 & 19.31 \\
\hline 13 & Chìsháo & red paeony root Radix Paeoniae Rubra ${ }^{2}$ & 44 & 18.88 \\
\hline 14 & Hónghuā & safflower Flos carthami (Hong-Hua) & 41 & 17.60 \\
\hline 15 & Báishù & largehead atractylodes rhizome Rhizoma atractylodis macrocephalae $e^{0,1}$ & 40 & 17.17 \\
\hline 16 & Chāngshù & Chinese atractylodes rhizome Rhizoma Aractylodis Lanceae ${ }^{0, \mathrm{f}}$ & 39 & 16.74 \\
\hline 17 & Shuǐzhì & hirudo leech Hirude nipponica Whitman & 37 & 15.88 \\
\hline 18 & Táorén & peach seed Semen Persicae & 37 & 15.88 \\
\hline 19 & Zéxiè & oriental waterplantain rhizome Rhizoma alismatis 0,1 & 36 & 15.45 \\
\hline 20 & Báisháo & white peony root Radix paeoniae alba ${ }^{0,1}$ & 36 & 15.45 \\
\hline 21 & Shúdihuáng & radix rehmanniae preparate Radix rehmanniae praeparata ${ }^{0,2}$ & 35 & 15.02 \\
\hline 22 & Màidōng & dwarf lilyturf tuber Radix ophiopogonis ${ }^{1}$ & 35 & 15.02 \\
\hline 23 & Zhīmŭ & common anemarrhena rhizom Rhizoma Anemarrhenae ${ }^{2}$ & 34 & 14.59 \\
\hline 24 & Gāncăo & licorice root Radix glycyrrhizae & 32 & 13.73 \\
\hline 25 & Huánglián & Coptic root Rhizoma coptidis ${ }^{0,1}$ & 30 & 12.88 \\
\hline 26 & Mŭdānpí & tree peony bark Cortex Moutan ${ }^{0}$ & 30 & 12.88 \\
\hline 27 & Chuānniúxī & medicinal cyathula root Radix cyathulae & 30 & 12.88 \\
\hline 28 & Dăngshēn & flase asiabeli root Radix Codonopsis Pilosulae ${ }^{2}$ & 29 & 12.45 \\
\hline 29 & Yìmŭcăo & motherwort herb Herba Leonuri & 29 & 12.45 \\
\hline 30 & Tàizĭshēn & heterophylly faalsestarwort root Radix pseudosteliariae & 27 & 11.59 \\
\hline 31 & Jīxuèténg & spatholobus suberectus dunn Caulis spatholobi & 27 & 11.59 \\
\hline 32 & Huángjīng & manyflower solomonseal rhizome Rhizoma polygonati $^{2}$ & 26 & 11.16 \\
\hline 33 & Dilóng & earthworm Lumbricus rubellus & 26 & 11.16 \\
\hline 34 & Dàhuång & rhubarb root Radix et rhizoma rhei & 22 & 9.44 \\
\hline 35 & Wŭwèizì & orange magnoliavine fruit Fructus Schisandrae Sphenantherae ${ }^{2}$ & 21 & 9.01 \\
\hline 36 & Shānzhā & hawthorn fruit Fructus Crataegi Cuneatae & 17 & 7.30 \\
\hline 37 & Sānq̄ī & radix notoginseng Panax notoginseng & 17 & 7.30 \\
\hline 38 & Jiāngcán & stiff silkworm Bombyx Batryticatus & 16 & 6.87 \\
\hline 39 & Jīnèijīn & chicken's gizzard-membrane Endothelium Coreneum Gigeriae Galli & 15 & 6.44 \\
\hline 40 & Cháihú & Chinese thorowax root Radix Bupleuri ${ }^{2}$ & 14 & 6.01 \\
\hline 41 & Guìzhī & cassia twig Ramulus Cinnamomi & 14 & 6.01 \\
\hline 42 & Rénshēn & ginseng Radix ginseng & 14 & 6.01 \\
\hline 43 & Shúfùzî & common monkshood root Radix aconiti lateralis preparata & 14 & 6.01 \\
\hline 44 & Yìyĭrén & jobstears seed Semen Coicis & 13 & 5.58 \\
\hline 45 & Yùzhú & fragrant solomonseal rhizome Rhizoma polygonati odorati & 13 & 5.58 \\
\hline 46 & Tùsīzì & Chinese dodder seed Cuscuta chinensis Lam & 13 & 5.58 \\
\hline 47 & Héshŏuwū & tuber fleeceflower root Radix polygoni multiflori & 12 & 5.15 \\
\hline 48 & Shāshēn & upright ladybell root Radix Adenophorae & 11 & 4.72 \\
\hline 49 & Chénpí & tangerine peel Pericarpium Citri Reticulatae & 11 & 4.72 \\
\hline 50 & Huángbăi & amur corktree bark Cortex Phellodendri & 11 & 4.72 \\
\hline 51 & Digŭpí & Chinese wolfberry root-bark Cortex Lycii & 11 & 4.72 \\
\hline 52 & Wüméi & dark plum fruit Fructus mume & 10 & 4.29 \\
\hline 53 & Shíhú & dendrobium Herba dendrobii & 9 & 3.86 \\
\hline 54 & Jīnyínhuā & honeysuckle flower Flos lonicerae & 9 & 3.86 \\
\hline 55 & Ròguì & cassia bark Cortex cinnamoni & 8 & 3.43 \\
\hline 56 & Zhīshí & immature orange fruit Fructus Aurantii Immaturus & 8 & 3.43 \\
\hline 57 & Yínyánghè & shorthorned epimedium herb Herba epimedii & 8 & 3.43 \\
\hline 58 & Guîjiànyŭ & winged euonymus twig Ramulus Euonymi & 8 & 3.43 \\
\hline 59 & Zélán & hirsute shiny bugleweed herb Herba lycopi & 8 & 3.43 \\
\hline 60 & Shārén & villous amomum fruit Fructus amomi & 8 & 3.43 \\
\hline 61 & Bànxià & pinellia tuber Rhizoma pinelliae & 8 & 3.43 \\
\hline 62 & Chēqiánzĭ & plantain seed Semen plantaginis & 8 & 3.43 \\
\hline 63 & Mùguā & common floweringquince fruit Fructus chaenomelis & 8 & 3.43 \\
\hline 64 & Mòyào & myrrh Commiphora myrrha & 8 & 3.43 \\
\hline 65 & Xixīn & manchurian wildginger Herba asari & 7 & 3.00 \\
\hline 66 & Mùxiāng & common aucklandia root Radix aucklandiae & 7 & 3.00 \\
\hline 67 & Yùjīn & aromatic turmeric root-tuber Radix curcumae aromaticae & 7 & 3.00 \\
\hline 68 & Hüzhàng & giant knotweed rhizome Rhizoma polygoni cuspidati & 7 & 3.00 \\
\hline 69 & Nüzhēzĭ & glossy privet fruit Fructus Ligustri Lucidi & 7 & 3.00 \\
\hline 70 & Ròucóngróng & desertliving cistanche Herba cistanches & 7 & 3.00 \\
\hline 71 & Rŭxiāng & frankincense Boswellia carterii & 7 & 3.00 \\
\hline 72 & Lìzhīhé & lychee seed Semen litchi & 6 & 2.58 \\
\hline 73 & Xīyángshēn & American ginseng Radix Panacis Quinquefolii & 6 & 2.58 \\
\hline 74 & Shēngjiāng & ginger peel Zingiber officinale Roscoe & 6 & 2.58 \\
\hline 75 & Zhìgāncăo & prepared liquorice root Radix Glycyrrhizae Preparata & 6 & 2.58 \\
\hline 76 & Sāngjìshēng & parasitic loranthus Loranthus parasiticus & 6 & 2.58 \\
\hline 77 & Sāngzhī & mulberry twig Ramulus Mori & 5 & 2.15 \\
\hline 78 & Sāngshèn & mrlberrt fruit Fructus Mori & 5 & 2.15 \\
\hline 79 & Xiāngfù & nutgrass galingale rhizome Rhizoma cyperi & 5 & 2.15 \\
\hline 80 & Zhìguībăn & tortoise shell Caraoax et Plastrum Testudinis & 5 & 2.15 \\
\hline 81 & Liánzĭròu & lotus seed Semen nelumbinis & 4 & 1.72 \\
\hline 82 & Zhìqiào & orange fruit Fructus Aurantii & 4 & 1.72 \\
\hline 83 & Kūnbù & kelp Thallus Laminariae et Eckloniae & 4 & 1.72 \\
\hline
\end{tabular}


Jie Yang. Journal of Diabetes Research and Clinical Metabolism 2012, http://www.hoajonline.com/journals/pdf/2050-0866-1-10.pdf

baikal skullcap root Radix scutellariae

white mulberry root-bark Cortex mori

gordon enryale seed Semen Euryales

largeleaf gentian root Radix Gentianae Macrophyllae

honeysuckle stem Caulis lonicerae

scorpion Scorpio

cassia seed Semen Cassiae

corn stigma Stigma Maydis

pod Bombyx mori Linnaeus

clematis root Radix Clematidis

twolobed officinal magnolia bark Cortex Magnoliae officinalis

cochinchinese asparagus root Radix Asparagi

gypsum Gypsum Fibrosuum

good days tea leaf Gynostemma pentaphyllum (Thunb.)Mak

akebia stem Caulis Akebiae

sargassum Sargassum pallidum

mantis egg-case Ootheca Mantidis

prepared common monkshood daughter root Radix Aconiti Lateralis Preparata zedoary Rhizoma Curcumae Curcuma zedoaria

yerbadetajo herb Herba ecliptae

white hyacinth bean Semen Lablab Album

agaric Polyporus

cattail pollen Pollen Typhae

cape jasmine fruit Fructus Gardeniae

snakegourd fruit Fructus Trichosanthis

chrysanthemum flower Flos chrysanthemi

perilla fruit Fructus Perillae

centipede Scolopendra

spina date seed Semen Ziziphi Spinosae Ziziphus jujuba Mill

dahurian angelica root Radix angelicae dahuricae

pedate pinallia Jackinthepulpit Rhizome

platycodon root Radix Platycodi

argy wormwood leaf Folium Artemisiae Argyi

lalang grass rhizome Rhizoma Imperatae

garden balsam stem Caulis Lonicerae

Chinense caterpillar fungus Cordycep

glandularstalk St. Herba SiegesbeckiaeSiegesbeckia orientalis L.

dandelion Herba taraxaci

Sappan wood Lignum sappan

fortune eupatorium herb Herba eupatori

Chinese eaglewood wood Lignum aquilariae sinensis

palmleaf raspberry fruit Fructus rubi

oyster shell Concha Ostreae

nacre concha Margaritifera Usta

common burreed rhizome Rhizoma Sparganii

parslane herb Herba Portulacae

hindu lotus leaf Folium Nelumbinis

great burdock achene Fructus Arctii

garden radish seed Semen Raphani Sativi

pancreas of pig

rose Flos Rosae Rugosae

philippine violet herb Herba Violae

Rhizoma Corydalis

common peony Paeonia lactiflora

tangerine pericarp Pericarpium

areca seed Semen arecae

medicated leaven Massa Medicata Fermentata

prince's feather fruit Fructus Polygoni Orientalis

largetrifoliolious bugbane rhizmome Rhizoma Cimicifugae

Chinese starjasmine stem Caulis Trachelospermi

notopterygium rhizome or root Rhizoma seu Radix Notopterygii

duhuo doubleteeth pubescent angelica root Radix Angelicae Pubescentis

ground beetle Eupolyphaga seu Steleophaga

white mustard seed Semen Sinapis Albae

dried ginseng Radix Ginseng

grassleaf sweetfalg rhizome Rhizoma Acoori Graminei

weeping forsythia capsule Fructus forsythiae

prepared pangolin scales Squama Manitis Preparata

driverse wormword herb Herba Artemisiae Anomalae

ephedra Herba Ephedrae

bitter apricot seed Semen Armeniacae Amarum

unibract fritillary bulb Bulbus Fritillariae Cirrhosae

teasel root Radix dipsaci

Chinese gall Galla Chinensis

sharpleaf glangal fruit Fructus Alpiniae Oxyphyllae

peppermint Mentha spicata

jujube Fructus Jujubae

willow Salix matsudana Koidz

medicinal indianmulberry root Radix Morindae Officinalis

lanceleaf lily bulb Bulbus Lilii

kadsura pepper stem Caulis Piperis Kadsurae

malaytea scurfpea fruit Fructus Psoraleae

Japanese felt fern Folium Pyrrosiae

anber Succinum

common monkshood mother root Radix Aconiti

glutinosae rice Oryzae Glutinosae

donkey-hide gelatin Colla corii asini ass

wheat bran Fructus Tritici Levis

medcinal evodia fruit Fructus Evodiae

1.72

1.72

1.72

1.72

1.72

1.72

1.72

1.72

1.72
1.72
1.72

1.29

1.29

1.29
1.29

(.29)

1.29

1.29

1.29
1.29

1.29

1.29

1.29

1.29

1.29

1.29
1.29

1.29

1.29
1.29

1.29

1.29

1.29

1.29

1.29

1.29

1.29

1.29

1.29

1.29

1.29

1.29

0.86

0.86

0.86

0.86

0.86

0.86

0.86

0.86

0.86

0.86

0.86

0.86

0.86

0.86
0.86

0.86

0.86

0.86
0.86

0.86

0.86

0.86
0.86

0.86

0.86

0.86

0.86

0.86

0.86

0.86

0.86

0.86

0.86

0.86

0.86

0.86

0.86

0.86

0.86

0.86

0.86

0.86

0.86

0.86

0.86

0.86

0.86

0.86

0.86

0.86

0.86

0.86

0.86

0.43

0.43
0.43 
Jie Yang. Journal of Diabetes Research and Clinical Metabolism 2012, http://www.hoajonline.com/journals/pdf/2050-0866-1-10.pdf

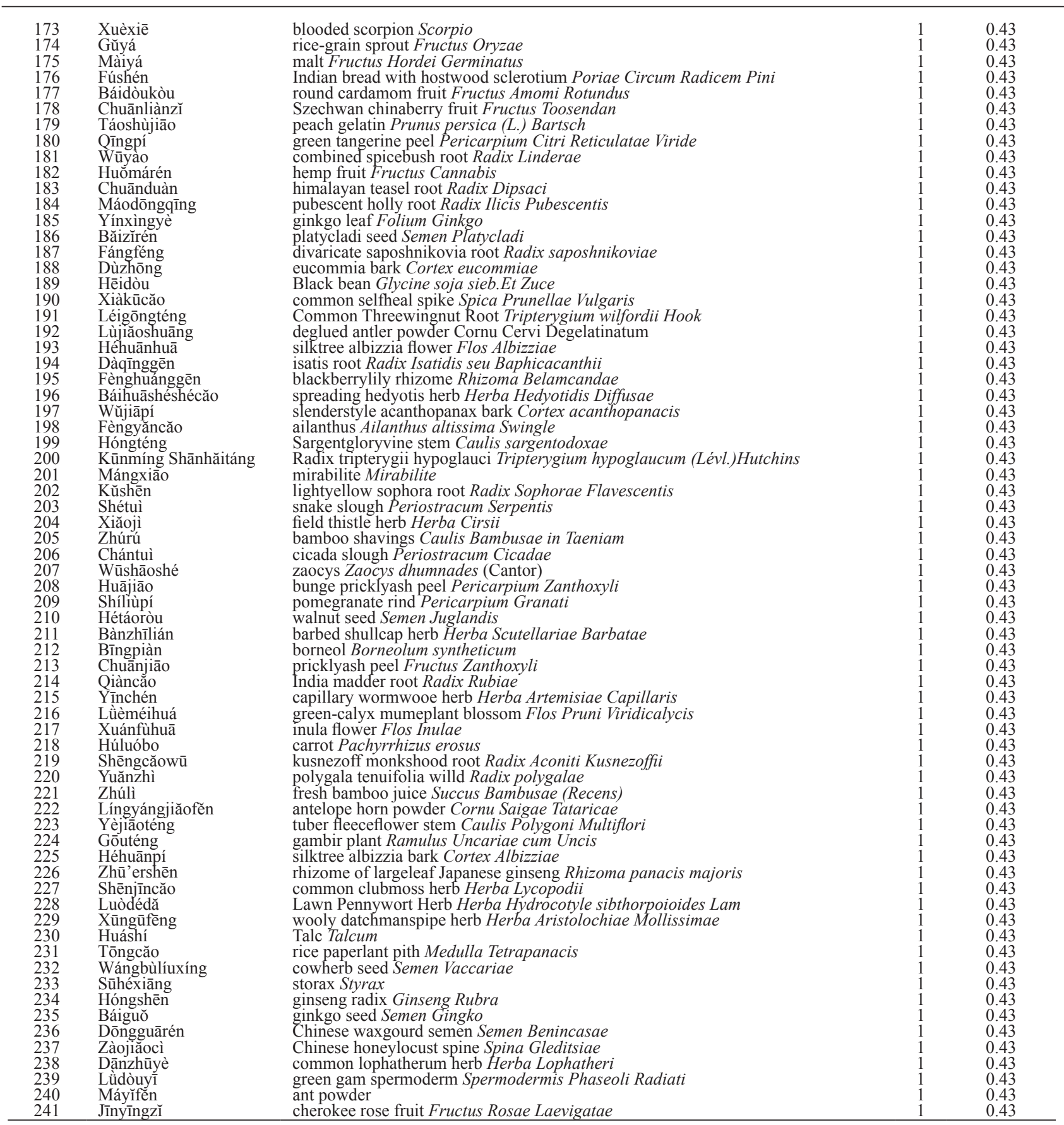

Note: * There are 241 species of Chinese medicinal materials (CMM) in diabetes Chinese traditional descriptions from a book named Diabetes Chinese Medicinal Proved Recipes (18). There are named by English andLatin name in order

for each herb. $c_{k}$ and $P_{k}$ mean the accumulative times and the percentage of each herb in 233 DCTPs, respectively.

“0” figures 18 kinds of Chinese tradicinal herbs consisting of the score matrix in the simple matrix method; " " and “2” express 10 kinds of Chinese tradicinal herbs consisting of the first score matrix and 12 kinds of Chinese tradicinal herbs consisting of the secondary score matrix in the complex matrix method, respectively. 
Table 2. The diabetes Chinese traditional prescriptions in training set and their compositions of the simple or complex score matrix method (16)

\begin{tabular}{|c|c|c|c|c|c|c|c|}
\hline \multirow{2}{*}{ No. } & \multirow{2}{*}{ Name } & \multirow{2}{*}{ Composition } & \multirow{2}{*}{$\begin{array}{c}\text { Clinical } \\
\text { efficiency }\end{array}$} & \multicolumn{2}{|c|}{$\begin{array}{l}\text { Simple matrix } \\
\text { method }\end{array}$} & \multicolumn{2}{|c|}{$\begin{array}{l}\text { Complex ma- } \\
\text { trix method }\end{array}$} \\
\hline & & & & $A$ & $P_{1}(\%)$ & $A$ & $P_{2}(\%)$ \\
\hline 1 & $\begin{array}{l}\text { Jian-Pi-San- } \\
\text { Jing-Tang }\end{array}$ & $\begin{array}{l}\text { membranous milkvetch root, lobed kudzurine root, Chinese atractylodes } \\
\text { rhizome, white mulberry root-bark, tuckahoe dried sclerotium, common } \\
\text { yam rhizome, lychee seed, Chinese thorowax root }\end{array}$ & $100 \%$ & 28 & 56 & 21 & 84 \\
\hline 2 & $\begin{array}{l}\text { San-Shen-Qi- } \\
\text { Shao-Tang }\end{array}$ & $\begin{array}{l}\text { membranous milkvetch root, heterophylly faalsestarwort root, upright } \\
\text { ladybell root, common yam rhizome, dwarf lilyturf tuber, asiatic cornelian } \\
\text { cherry fruit, fllase asiabeli root, white peony root, orange magnoliavine } \\
\text { fruit, dark plum fruit, Chinese atractylodes rhizome }\end{array}$ & $93 \%$ & 27 & 54 & 20 & 80 \\
\hline 3 & Xin-Run-Fang & $\begin{array}{l}\text { membranous milkvetch root, Chinese dodder seed, common yam rhizome, } \\
\text { radix rehmanniae preparata, common anemarrhena rhizom, lotus seed, } \\
\text { manchurian wildginger, cassia bark }\end{array}$ & $96.67 \%$ & 20 & 40 & 17 & 68 \\
\hline 4 & $\begin{array}{l}\text { Jiang-Tang- } \\
\text { Jiao- Lang }\end{array}$ & $\begin{array}{l}\text { ginseng, tuckahoe dried sclerotium, membranous milkvetch root, largehead } \\
\text { atractylodes rhizome, rehmannia dried rhizome, figwort root, lobed kudzu- } \\
\text { rine root, common yam rhizome, licorice root, mongolian snakegourd root, } \\
\text { manyflower solomonseal rhizome }\end{array}$ & $87.8 \%$ & 38 & 76 & 22 & 88 \\
\hline 5 & $\begin{array}{l}\text { Jia-Wei-Shen- } \\
\text { Qi-Wan }\end{array}$ & $\begin{array}{l}\text { radix rehmanniae preparata, common yam rhizome, asiatic cornelian cherry } \\
\text { fruit, tree peony bark, tuckahoe dried sclerotium, oriental waterplantain rhi- } \\
\text { zome, common monkshood root, cassia bark, membranous milkvetch root, } \\
\text { fllase asiabeli root, lobed kudzurine root, largehead atractylodes rhizome }\end{array}$ & $91.38 \%$ & 37 & 74 & 22 & 88 \\
\hline 6 & $\begin{array}{l}\text { Si-Miao-San- } \\
\text { Fang }\end{array}$ & $\begin{array}{l}\text { Chinese atractylodes rhizome, achyranthes root, jobstears seed, amur cork- } \\
\text { tree bark, fortune eupatorium herb, chicken's gizzard-membrane, danshen } \\
\text { root, lychee seed, Coptic root }\end{array}$ & $97.5 \%$ & 10 & 20 & - & - \\
\hline 7 & $\begin{array}{l}\text { Xiao-Ke-Jiang- } \\
\text { Tang-Jiao-Lang }\end{array}$ & $\begin{array}{l}\text { membranous milkvetch root, figwort root, rehmannia dried rhizome, com- } \\
\text { mon anemarrhena rhizom, dwarf lilyturf tuber, lycium barbarum fruit, com- } \\
\text { mon yam rhizome, asiatic cornelian cherry fruit, fllase asiabeli root, orange } \\
\text { magnoliavine fruit, baikal skullcap root, Chinese wolfberry root-bark, } \\
\text { danshen root, licorice root }\end{array}$ & $89.4 \%$ & 34 & 68 & - & - \\
\hline 8 & $\begin{array}{l}\text { Zi-Yin-Yi-Shen- } \\
\text { Tang }\end{array}$ & $\begin{array}{l}\text { mongolian snakegourd root, dendrobium, common yam rhizome, radix } \\
\text { rehmanniae preparata, dwarf lilyturf tuber, glossy privet fruit, yerbadetajo } \\
\text { herb, parasitic loranthus, membranous milkvetch root, white peony root, } \\
\text { common anemarrhena rhizom, twotoothed achyranthes root Radix achyran- } \\
\text { this bidentatae (Niúxī), licorice root }\end{array}$ & $89.3 \%$ & 26 & 52 & - & - \\
\hline 9 & Xiao-Ke-Fang & $\begin{array}{l}\text { rehmannia dried rhizome, heterophylly faalsestarwort root, common yam } \\
\text { rhizome, danshen root, lycium barbarum fruit, palmleaf raspberry fruit }\end{array}$ & $100.00 \%$ & - & - & 16 & 64 \\
\hline 10 & $\begin{array}{l}\text { Jia-Wei-Huang- } \\
\text { Lian-Ajiao-Tang }\end{array}$ & $\begin{array}{l}\text { Coptic root, baikal skullcap root, radix notoginseng, donkey-hide gelatin, } \\
\text { white peony root, danshen root, mongolian snakegourd root, common } \\
\text { anemarrhena rhizom, licorice root }\end{array}$ & $91.00 \%$ & - & - & 15 & 60 \\
\hline 11 & $\begin{array}{l}\text { Zi-Yin-Run-Zao- } \\
\text { Huo-Xue-Tang }\end{array}$ & $\begin{array}{l}\text { membranous milkvetch root, figwort root, rehmannia dried rhizome, } \\
\text { danshen root, manyflower solomonseal rhizome, dwarf lilyturf tuber, red } \\
\text { paeony root }\end{array}$ & $100.00 \%$ & - & - & 20 & 80 \\
\hline
\end{tabular}

Note: $P_{1}$ and $P_{2}$ mean the percentage of the total score ( ) of some proved recipe to the score standard of training set based on the simple or complex score matrix method.

total score $(A)$ of some pending proved recipe is composed of two parts as follows: $A=\sum\left(q_{j}\right) q_{j}=\left\{\begin{array}{ll}\sum_{j=1}^{n}\left(s_{j}\right) & (j \in i) \\ 0 & (j \notin i)\end{array}\right.$. And take the percentage of the total score ( $A$ ) of some proved recipe to the score standard of training set for evaluation the pending recipes, which is generally figured as follows: $P_{2}=A / M \times 100 \%$.

\section{Experimental Results}

\section{Analysis on diabetes mellitus proved recipe}

Table 1 lists the accumulative total times and percentage of 241 kinds of CMMs occurring in DMPRs. And membranous milkvetch root has a high percentage of up to $65.66 \%$ and danshen root is $50.64 \%$. There are 31 kinds of CMMs with a percentage of more than $10 \%$, which are common yam rhizome, rehmannia dried rhizome, Chinese angelica, lobed kudzurine root, asiatic cornelian cherry fruit, mongolian 
Table 3. The approximate scores of corresponding herbs in the first score matrix of the complex matrix method based on drug pairs

\begin{tabular}{|c|c|c|c|}
\hline $\begin{array}{l}\text { Name of herbs in the first score } \\
\text { matrix }\end{array}$ & $\begin{array}{l}\text { Scores in the } \\
\text { first score matrix }\left(S_{1}\right)\end{array}$ & Name of corresponding herbs & $\begin{array}{l}\text { Scores (integral) } \\
\qquad\left(S_{2}\right)^{*}\end{array}$ \\
\hline & & common yam rhizome & 4 \\
\hline membranous milkvetch root & 6 & lobed kudzurine root & 3 \\
\hline danshen root & 4 & mongolian snakegourd root & 2 \\
\hline rehmannia dried rhizome & 3 & radix rehmanniae preparata & 1 \\
\hline Chinese atractylodes rhizome & 2 & figwort root & 2 \\
\hline & & manyflower solomonseal rhizome & 1 \\
\hline largehead atractylodes rhizome & 2 & $\begin{array}{l}\text { fllase asiabeli root } \\
\text { lycium barbarum fruit }\end{array}$ & $\begin{array}{l}1 \\
1\end{array}$ \\
\hline asiatic cornelian cherry fruit & 2 & orange magnoliavine fruit & 1 \\
\hline dwarf lilyturf tuber & 2 & $\begin{array}{l}\text { common anemarrhena rhizom } \\
\text { red paeony root }\end{array}$ & $\begin{array}{l}2 \\
2\end{array}$ \\
\hline white peony root & 2 & Chinese thorowax root & 1 \\
\hline
\end{tabular}

Note: * conversion as follows: $S_{2}=s_{1} \times \frac{c_{k p}}{c_{k p^{\prime}}}, S_{1}$ and $S_{2}$ express the score $\left(S_{i}\right)$ of each herb in first matrix and the relative score of each corresponding herb of drug pairs in secondary matrix (integer), respectively; $C_{k p}$ and $C_{k p}$, figure the accumulative times of a group of drug pairs tagged by subscripts ${ }_{p}$ and ${ }_{p^{\prime}}$ (Table 1), and the former for the secondary matrix and the later for the first matrix.

snakegourd root, szechwan lovage rhizome, tuckahoe dried sclerotium, figwort root, lycium barbarum fruit, red paeony root, safflower, largehead atractylodes rhizome, Chinese atractylodes rhizome, hirudo leech, peach seed, oriental waterplantain rhizome, white peony root, radix rehmanniae preparata, dwarf lilyturf tuber, common anemarrhena rhizom, licorice root, danshen root, Coptic root, tree peony bark, medicinal cyathula root, motherwort herb, heterophylly faalsestarwort root, spatholobus suberectus dunn, manyflower solomonseal rhizome, and earthworm, in turn; while there are 19 kinds of $\mathrm{CMHs}$ more than $4 \%$, namely rhubarb root), orange magnoliavine fruit, hawthorn fruit, radix notoginseng, stiff silkworm, chicken's gizzard-membrane, Chinese thorowax root, cassia twig, ginseng, common monkshood root, jobstears seed, fragrant solomonseal rhizome, Chinese dodder seed, tuber fleeceflower root, upright ladybell root, tangerine peel, amur corktree bark, Chinese wolfberry root-bark, and dark plum fruit; and the others have lower percent, in turn.

\section{Simple score matrix and evaluation of CDMR}

On the basis of our methods [18], a training set (Table 2) is composed of eight DMPRs, namely Jian-Pi-San-Jing-Tang, San-Shen-Qi-Shao-Tang, Xin-Run-Fang, Si-Miao-San-Fang, Jiang-Tang-Jiao-Lang, Jia-Wei-Shen-Qi-Wan, Zi-Yin-Yi-Shen-Tang, and Xiao-Ke-Jiang-Tang-Jiao-Lang, which contain 57 kinds of CMMs. According to the order of 241 kinds of CMMs, the matching direction of medicinal herbs, and the character of drug pairs (mated herbs), choose 18 kinds of CMM for a $1 \times 18$ unitary score matrix consisting of zeros for scoring mismatches and a series of positive scores namely (7 733333333222 21111 ) on the diagonal for scoring 18 kinds of CMM match with each other, which are membranous milkvetch root, common yam rhizome, figwort root, danshen root, Chinese atractylodes rhizome, lobed kudzuvine root, tuckahoe dried sclerotium, asiatic cornelian cherry fruit, rehmannia dried rhizome, radix rehmanniae preparata, white peony root, largehead atractylodes rhizome, Mongolian snakegourd root, tree peony bark, oriental waterplantain rhizome, Coptic root, Chinese angelica, and lycium barbarum fruit in turn.

\section{Complex score matrix and evaluation of CDMR}

Similarly, a training set (Table 2) is composed of eight DMPRs, namely Jian-Pi-San-Jing-Tang, San-Shen-Qi-Shao-Tang, XinRun-Fang, Jiang-Tang-Jiao-Lang, Xiao-Ke-Fang, Jia-Wei-ShenQi-Wan, Jia-Wei-Huang-Lian-Ejiao-Tang, and Zi-Yin-Run-ZaoHuo-Xue-Tang. Choose 10 kinds of CMMs consisting of a first score matrix $1 \times 10$ consisting of zeros for scoring mismatches

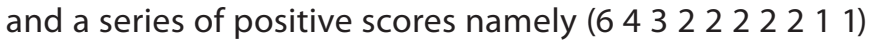
on the diagonal, which are membranous milkvetch root, danshen root, rehmannia dried rhizome, dwarf lilyturf tuber, asiatic cornelian cherry fruit, Chinese atractylodes rhizome, white peony root, largehead atractylodes rhizome, oriental waterplantain rhizome, and Coptic root, in turn.

Considering that some drug pairs play important roles in treating diabetes, introduce some mated herbs to first score matrix to construct a secondary score matrix. These drug pairs include yin-nourishing and qi-tonic mated herbs and yang-tonic mated herbs. The former involves membranous milkvetch root and common yam rhizome, membranous milkvetch root and lobed kudzurine root, danshen root and mongolian snakegourd root, Chinese atractylodes rhizome and figwort root, figwort root and mongolian snakegourd root, 
largehead atractylodes rhizome and manyflower solomonseal rhizome, and asiatic cornelian cherry fruit complex with lycium barbarum fruit and orange magnoliavine fruit. The latter, such as asiatic cornelian cherry fruit and lycium barbarum fruit, danshen root complex with tree peony bark and red paeony root, and Chinese thorowax root complex with red paeony root and white peony root, could invigorate liver and kidney to activate yang, circulate blood, and boost bones and muscles to strengthen the body.

On the basis of the first score matrix, we thereby convert the score values of another corresponding herb in drug pairs (Table 3) as follows: $s_{2}=s_{1} \times \frac{c_{k p}}{c_{k p^{\prime}}}, C_{k p}$ and $C_{k p}$, figure the accumlative times of a group of drug pairs tagged (Table 1). Take membranous milkvetch root for example whose $S_{1}$ and $C_{k p^{\prime}}$ values are 6 and 153. There are two corresponding herbs to it, namely common yam rhizome and lobed kudzurine root. Here, their $C_{k p}$ values are 97 and 67, respectively. So the $S_{2}$ values of common yam rhizome and lobed kudzurine root are integrally 4 and 3, respectively, namely $s_{2}=6 \times \frac{97}{153} \approx 4$ and $s_{2}=6 \times \frac{67}{153} \approx 3$. The rest may be deduced by analogy. And then obtain a secondary score matrix $1 \times 12$ consisting of zeros for scoring mismatches and a series of positive scores namely (4 322221111111 ) on the diagonal for scoring 12 kinds of CMM match with each other, which are common yam rhizome, lobed kudzurine root, mongolian snakegourd root, red paeony root, common anemarrhena rhizom, figwort root, lyceum barbarum fruit, orange magnoliavine fruit, Chinese thorowax root, radix rehmanniae preparata, manyflower solomonseal rhizome, and fllase asiabeli root, in turn.

\section{Statistic analysis}

Results reveal that the predicting results and their clinical effects don't exhibit linear relationship but show prediction diversity (Figure 1). The exceptive instances with $P$ value of more than $100 \%$ go beyond the range of $P$ value in bioinformatics $(0 \leq P \leq 1)$, because the complementarity of the secondary score matrix make the prediction score largen but evaluate standard still adopts the score standard of the first score matrix. The instances with $P$ value of less than $50 \%$, however, rifely occur to those possessing good therapy effect. This is because those CMMs in score matrixs exist in these DMPRs with a lower probability. Similarly, due to not containing those CMMs in score matrixs, approximate 10 kinds of DMPRs still have wonderful treatment effect notwithstanding their $P$ values equal to zero. Figure 1 presents the comparison between prediction values with matrix comparison and clinical effects of 225 kinds of DMPRs.

The $\mathrm{t}$-test results reveal that $\mathrm{t}$ value is $\mathbf{1 1 . 5 2 4}$ more than 3.291 $\left(\mathrm{t}_{0.001}\right)$, which indicates that the difference between the average $P$ values of two methods displays a remarkable significance. It is that $P_{2}$ values and $P_{1}$ values show observably difference, and the complex matrix method

$(x \pm s(n)=73.25 \pm 27.93(225))$ is more logical and more

credible than the simple matrix method

$(\bar{x} \pm s(n)=46.24 \pm 21.37(225))$. The complex matrix

method possesses higher prediction values and more reliability than the simple matrix method. Figure 2 displays the distributing map of $P_{2}$ values of 225 kinds of Chinese diabetes medicinal recipes, which percents definite normal distribution. Moreover, the range between 45.32 and 101.18 distributes 167 samples with a percentage of up to $74.22 \%$. Without deflective samples, the $P_{2}$ values of 167 samples show more concentricity than their $P_{1}$ values, namely $x \pm s(n)=77.35 \pm 14.96(167)$.

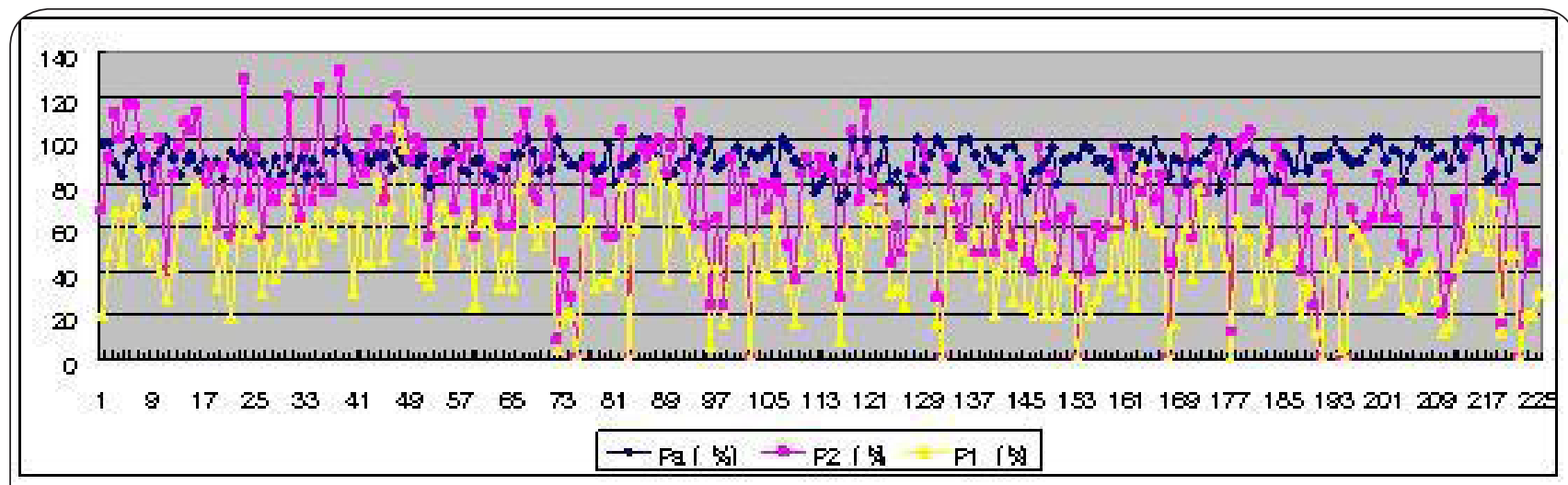

Figure 1. Comparison with the predicted values of 225 kinds of Chinese diabetes meidicinal recipes and their clinical results. The rhombic dots (blue), triangular dots (yellow), and quadrate dots (red) denote the clinic results, the predicted values of the simple matrix method, and the predicted values of the complex matrix method, respectively. 


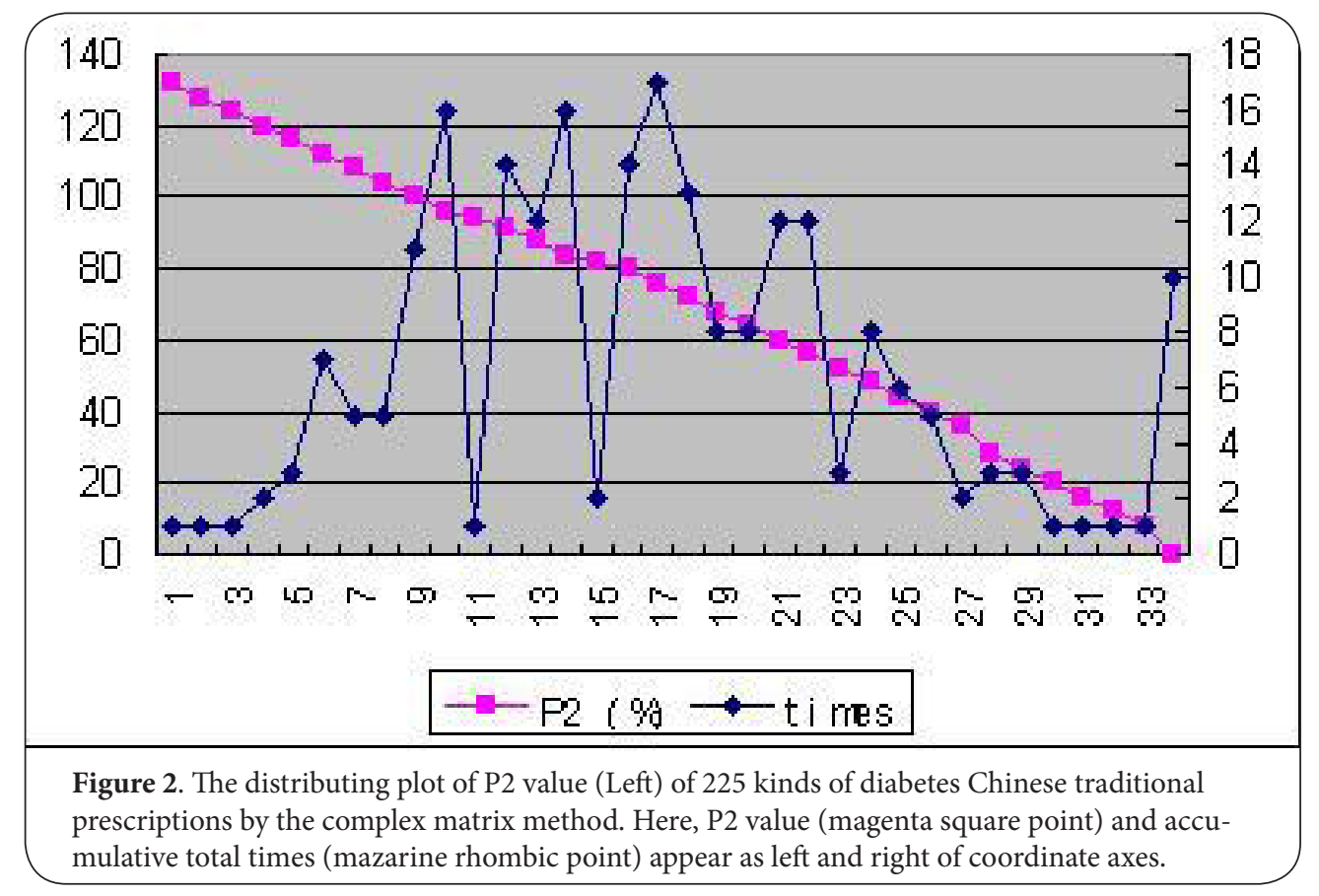

\section{Discussion}

Chinese medicine is a bright phearl of worldwide trandicinal medicine mine whose hardcore comprises some group of dialectic relationships, such as Zang and Fu, Jing (longitude points) and Luo (latitude points), jing (liquid) and qi (gas), jin (saliva) and xue (blood), etc. Chinese medicine considers that Zang and Fu actually indicate the two aspects opposite to each other of the same object. The relation of Zang and Fu corresponds to yin and yang relation as well as $L i$ (the inside) and Biao (the outside) relation; and Zang denotes negative viscera, such as heart, lung, pancreas, liver, etc, which behave inside while $\mathrm{Fu}$ shows corresponding positive and outside matter. Zang and Fu form interactive Luo (latitude points) resembling nets by Jing pusle (longitude points). And diabetes is categorized as Xiāokëzhèng (in Chinese pinyin) of Chinese medicine and undergoes a long course of three phages: yin deficiency, the coexistence of yin deficiency and drynessheat, and diabetes complications. Chinese medicine theory regards the weakness and akinesia of spleen as the essence of diabetes. Diabetes doesn't finally been cured with yin weakness, impetuous dryness-heat, and blood agglomeration all the time. Clinically, the traditional treatment of diabetes in Chinese medicine is based on the principle of eliminating heat by nourishing yin, moistening dryness and promoting fluid production. However, the current treatment in Western medicine mainly focuses on improving body sensitivity to insulin, correcting metabolic turbulence, researching the cause of formation insulin resistance.
Analysis of diabetes Chinese traditional prescriptions

According to the condition of the principal and secondary aspects, deficiency and excess, as well as the location of pathological changes of the disease, the following methods are respectively adopted: clearing heat and purging fire, resolving phlegm to activate meridians, promoting blood circulation to remove blood stasis, removing dampness, nourishing the kidney to replenish yin, invigorating the spleen to tonify qi, replenishing both yin and yang, etc. Based on such a medical opinion, a general therapeutical rule is to promote blood circulation to remove blood stasis, and secondly to activate vital energy circulation, to clear away heat in the body, to invigorate liver and kidney to activate yang, and to invigorate the spleen and stomach to strengthen the body [6]. The treatment of diabetes mostly adopts the following fundamental methods through the all remedial process, namely invigorating the spleen and nourishing the kidney method, ascending qing (lucidity) and converting zhuo (turbidity) method, benefiting qi and nourishing yin method, and circulating blood and dissolving blood stasis method [16]. Moreover, we found that DMPRs are mostly composed of the following CMMs classified as seven types: i) protecting spleen and benefiting kidney CMMs (such as membranous milkvetch root, common yam rhizome, asiatic cornelian cherry fruit, and mongolian snakegourd root); ii) nourishing yin and restoring kidney CMMs (manyflower solomonseal rhizome, figwort root, rehmannia dried rhizome, common yam rhizome, and radix rehmanniae preparata); iii) nourishing yin and yielding jin (saliva liquid) CMMs (common anemarrhena 
rhizom, lobed kudzurine root, mongolian snakegourd root rehmannia dried rhizome, and hirudo); iv) tonifying liver and spleen CMMs (common yam rhizome, asiatic cornelian cherry fruit, tuckahoe dried sclerotium, tree peony bark, and oriental waterplantain rhizome); v) circulating blood and dissolving gore CMMs (danshen root, rehmannia dried rhizome, Chinese angelica, szechwan lovage rhizome, safflower, hirudo, peach seed, medicinal cyathula root, spatholobus suberectus dunn, earthworm, rhubarb root, radix notoginseng, stiff silkworm, chicken's gizzard-membrane, tree peony bark, white peony root, motherwort herb, winged euonymus twig); vi) tonifying and invigorating liver CMMs (Chinese thorowax root, white peony root, motherwort herb, red paeony root, nutgrass galingale rhizome, rehmannia dried rhizome, danshen root, and fragrant solomonseal rhizome); vii) removing dampness CMMs (Chinese atractylodes rhizome, tuckahoe dried sclerotium, Coptic root, and clematis root); etc. And these CMMs possess good clinical effect. Furthermore, safflower, peach seed, and slenderstyle acanthopanax bark could circulate blood, dissolve gore, expand micro-vessel, and ameliorate local supply of blood and oxygen.

At the same time, we find some lowering-glucose drug pairs appearing in DMPRs [16], such as a couple of membranous milkvetch root and common yam rhizome, Chinese atractylodes rhizome and figwort root, membranous milkvetch root and lobed kudzurine root, danshen root and mongolian snakegourd root, danshen root and lobed kudzurine root, etc. The first two play a role of protecting spleen to converge jin, and nourishing yin to descend fire. Membranous milkvetch root coupled with common yam rhizome could restore spleen and consolidate kidney to ascend yang. Membranous milkvetch root tastes sweet to recruits $q i$, and ascends and restores spleen, while common yam rhizome restores spleen and cultivates lung to nourish yin and to yield jin, which could replenish yang and yin, nourish spleen and stomach, and promote transportation and translation. The midst two could reduce blood glucose and urine glucose, and the lobed kudzurine root of them can help membranous milkvetch root to ascend spleen. The last one could also reduce blood glucose and blood lipid, and could availably prevent and treat diabetes and its complication, such as hypertension, high blood lipid, and arteriosclerosis [16]. Sequentially, there are also some drug pairs possess reducing blood glucose activities. For example, a couple of figwort root and mongolian snakegourd root nourishs the kidney to replenish yin, clears away heat in the body, invigorate the stomach to strengthen the body, and reduces blood glucose; largehead atractylodes rhizome coupled with manyflower solomonseal rhizome invigorates the spleen to tonify qi, nourishs yin to moisten lung, enhances body immunity function, and reduces blood lipid and blood glucose; fllase asiabeli root and largehead atractylodes rhizome could help membranous milkvetch root to protect spleen, benefit qi and reduce blood glucose. In addition, both ginseng and common monkshood root could produce cortin-like effect and invigorate qi to strengthen the body. And ginseng coupled with common monkshood root mated herbs could supple each other and bring out the best in each other. Especially, ginseng is well known to be a good tonic for health care and many active constituents have been isolated and some preparations of ginseng have been developed and used in clinical treatment of diabetes in China. The extracts of all parts of ginseng (the roots, stems, leaves and fruits) are of anti-hyperglycemic effect $[20,21]$ such as ginsenoside, panaxans and ginseng polypeptides. Ginsenoside Rb1, Rg1, Re, Rg3, CEG, Rb2, CY, DPG-3-2 were isolated and experimentally or clinically confirmed to be bioactive for anti-diabetes or/and anti-diabetic complications. Panaxans A and B were also obtained from Radix Ginseng for anti-diabetes. The mechanism to lower blood glucose is to enhance insulin sensitivity, regulate the activity of enzymes related to glucose metabolism directly and/or indirectly [6]. On the other hand, we find some compound recipes existing in DMPRs, such as Liu-Wei-Di-Huang-Wan, Jin-Kui-Shen-QiWan, Fu-Shen-Wan, Fang-Ji-Huang-Qi-Tang, Sheng-Mai-San, Xiao-Ke-Wan, Yu-Quan-Wan, Si-Ni-San, Yu-Ye-Tang, Xio-Yao-San, Huang-Lian-Ejiao-Tang, Shen-Wu-Tang, Zhi-Bai-Di-HuangTang, San-Ren-Tang, Si-Mo-Tang, Si-Wu-Tang, Tao-Hong-Si-WuTang, and Xiao-Ke-Wu-Chong-Fang. For example, Liu-Wei-DiHuang-Wan comprises six kinds of tradicinal Chinese herbs, namely rehmannia dried rhizome, common yam rhizome, asiatic cornelian cherry fruit, oriental waterplantain rhizome, tuckahoe dried sclerotium, and tree peony bark, which nourishs the kidney to replenish yin, invigorates the spleen to tonify $q i$, and invigorates liver to activate yang. Jin-Kui-Shen-QiWan derives from the former with the addition of common monkshood root and cassia bark, lowering blood glucose and blood lipid with enhancing immunity function. Fu-Shen-Wan composes of Indian bread with hostwood sclerotium, grassleaf sweetfalg rhizome, polygala tenuifolia willd, tuckahoe dried sclerotium, and ginseng, while Fang-Ji-Huang-Qi-Tang is made of fourstamen stephania root, membranous milkvetch root, largehead atractylodes rhizome, licorice root, ginger peel, and jujube, and both of them invigorate the spleen to tonify qi, cleare water, and purge turgescence. Sheng-MaiSan, consisting of ginseng, dwarf lilyturf tuber and orange magnoliavine fruit, Xiao-Ke-Wan, made of lobed kudzurine root, rehmannia root, membranous milkvetch root, mongolian snakegourd root, corn stigma, orange magnoliavine fruit, common yam rhizome, and glibenclanmide, and Yu-QuanWan of lobed kudzurine root, mongolian snakegourd root, rehmannia root, dwarf lilyturf tuber, orange magnoliavine fruit, and licorice root, could invigorate the spleen to tonify $q i$, nourish the kidney to replenish yin, and clinically treat NIDDM. Si-Ni-San, Huang-Lian-Ejiao-Tang, Shen-Wu-Tang, etc, come from a Chinese ancient book named "Shang Han Lun (Dissertation of Typhoid)" written by Zhang Zhong-Jing, which possess preferable effect to treat type 2 diabetes. SiWu-Tang, consisting of radix rehmanniae preparata, Chinese 
angelica, white peony root, and szechwan lovage rhizome, Tao-Hong-Si-Wu-Tang pulsing two herbs of peach seed and safflower, and Xiao-Ke-Wu-Chong-Fang consisting of hirudo, stiff silkworm, scorpion, centipede, and zaocys, could promote blood circulation to remove blood stasis, reduce blood glucose, and treat diabetes and its complications [16]. Moreover, most of western medicines, which are often made of a single chemical compound, are very effective for directly relief of symptoms, such as lowering blood sugar. So, in the Chinese medical system, it is considered that the efficacy of almost all western medicines to lower blood glucose is better than Chinese traditional medicines but not good for diabetic complications. Chinese medicines are more effective not only to treat and prevent diabetic complications but also at the meantime to lower blood glucose level. Therefore, Chinese doctors often make a combination of traditional medicine with western medicine, western medicine for reducing blood sugar, traditional medicine for integrated care of body $[6,22]$. Our analysis results show that some three- or four-member CMM complexes have good effects reducing blood glucose, such as membranous milkvetch root complex with danshen root and rehmannia dried rhizome occurs in DMPRs with a high percentage of $23.61 \%$, membranous milkvetch root complex with rehmannia dried rhizome, and mongolian snakegourd root with $13.73 \%$, while membranous milkvetch root complex with danshen root, rehmannia dried rhizome, and mongolian snakegourd root with $10.30 \%$. And the percentages of the other four three-member complexes, namely membranous milkvetch root-rehmannia dried rhizome-lycium barbarum fruit, membranous milkvetch root-danshen root-lycium barbarum fruit, membranous milkvetch root-danshen root-Chinese atractylodes rhizome, and rehmannia dried rhizome-asiatic cornelian cherry fruit-lycium barbarum fruit, are $9.87 \%, 9.01 \%, 8.15 \%$, and $7.30 \%$, in turn. Danshen root complex with tree peony bark and red paeony root could promote blood circulation to remove blood stasis; whereas Chinese thorowax root-red paeony root-white peony root complex could invigorate liver to activate yang, promote blood circulation, and expedite Jing-Luo pulse. Some complexes, such as Asiatic cornelian cherry fruit complexed with Chinese dodder seed, lycium barbarum fruit complexed with Chinese dodder seed, and asiatic cornelian cherry fruit complexed with lycium barbarum fruit and orange magnoliavine fruit, could also effectively protect kindney.

Moreover, modern pharmacology research found that some $\mathrm{CMMs}$ possess notable lowering blood glucose effects, such as membranous milkvetch root, common anemarrhena rhizom, lobed kudzurine root, rehmannia dried rhizome, hirudo, danshen root, Chinese atractylodes rhizome, figwort root, Mongolian snakegourd root, dark plum fruit, asiatic cornelian cherry fruit, common yam rhizome, and radix rehmanniae preparata. And found that oriental waterplantain rhizome reduces blood lipid; Coptic root depresses blood pressure and blood glucose; radix notoginseng circulate blood and dissolve gore, and ameliorate microcirculation [16]. Further approved that ginseng, membranous milkvetch root, and mongolian snakegourd root not only promote beta-cell to be repaired, but also increase body insulin. Astragalus polysaccharides have an effect to two-dimensionally regulate the level of blood glucose, which can increase the blood glucose of hypoglycemic animals or humans to normal level, and significantly lower the level of blood glucose, triglyceride and myocardial calcium, improve the abnormalities of myocardial ultrastructure and the metabolism of diabetic rats and mice [23], and inhibit the onset of type 1 diabetes in nonobese diabetic mice [7]. Astragalus membranaceus (Huángqí) was reported to have an effect for diabetic complications, such as protecting the myocardium in diabetic nephropathy by inhibiting lipid peroxidation, prolonging the incubation period of late diabetic neuropathy by decreasing the motion nerve conduction velocity as an aldose reductase inhibitor, and exerting a beneficial effect on experimental diabetic nephropathy by suppressing the renal hypertrophy and microalbuminuria [24].

Of 241 kinds of Chinese medicinal materials, there are nine important CMMs for treating diabetes of type 2 in the early, middle, and terminal phases (such as membranous milkvetch root, danshen root, common yam rhizome, rehmannia dried rhizome, mongolian snakegourd root, lycium barbarum fruit, red paeony root, common anemarrhena rhizom, and manyflower solomonseal rhizome), four for the early and middle diabetes (figwort root, dwarf lilyturf tuber, Chinese atractylodes rhizome, and Coptic root), three for the early (lobed kudzurine root, tuckahoe dried sclerotium, and ginseng), six for the middle (Chinese angelica, szechwan lovage rhizome, motherwort herb, heterophylly faalsestarwort root, orange magnoliavine fruit, and fragrant solomonseal rhizome), and two for the terminal (cassia twig and Chinese dodder seed). Moreover, four CMMs (such as common yam rhizome, manyflower solomonseal rhizome, common anemarrhena rhizom, and rehmannia root) contain glucide or saccharide molecules, seven CMMs (membranous milkvetch root, ginseng, red paeony root, licorice root, oriental waterplantain rhizome, rehmannia root, and radix notoginseng) include terpene compounds besides osides molecules, two CMMs (danshen root and rhubarb root) contain quinone compounds, and five CMMs (Chinese atractylodes rhizome, largehead atractylodes rhizome, Chinese angelica, szechwan lovage rhizome, and orange magnoliavine fruit) contain sesquiterpene oils. And the liganans of orange magnoliavine fruit has protecting liver activity while Tannins of rhubarb root could reduce urea and protect kidney. Especially membranous milkvetch root has various functions, such as reducing blood pressure, antivirus, sterilization, composure, anticancer, and preventing coronary heart disease, besides reducing blood glucose.

Research result also reveals that some DMPRs possess good clinical effects in despite of whose $P$ value of less than $50 \%$ and equal to zero, which is because these prescriptions contain a few CMMs involved in score matrixs or none but there are a 
lot of other CMMs existing in these prescriptions. For example, there are a lot of CMMs promoting blood circulation to remove blood stasis (such as cyathula offficinalis kuan, spatholobus suberectus dunn, Chinese angelica, peach seed, dahurian angelica root, frankincense, myrrh, and honeysuckle stem), some CMMs strengthening the body (eucommia bark, teasel root, achyranthes root), and desertliving cistanche), some CMMs clearing heat and purging fire (honeysuckle flower, weeping forsythia capsule, chrysanthemum flower, giant knotweed rhizome, and dandelion), and some others (aromatic turmeric root-tuber, grassleaf sweetfalg rhizome, borneol, storax, Sappan wood, twolobed officinal magnolia bark immature orange fruit, Chinese eaglewood wood, tangerine peel, and nutgrass galingale rhizome), playing important roles in DMPRs for diabetes therapy though they don't belong to the score matrixs.

\section{Analysis of informatics}

Automated sequence comparisons based on sequence alignment are among the most familiar procedures in biological research, which is used to analysis of protein and of nucleic acid. People generally adopt amino acid sequence alignment and homology modeling to predict protein structure and function, including active sites, binding sites, domains, etc. Similarly, use nucleotide sequence alignment to analyze function motif of gene and to predict gene function [2]. In addition to database searches, pairwise and multiple alignment of sequences are often the starting points for in vitro mutagenesis, homology modeling and other procedures in molecular biology and biochemistry. All sequence-comparison methods require an alignment algorithm and a set of scores. Substitution scores quantify the cost of exchanging one residue for another, and gap penalties quantify the cost of exchanging a residue or a string of residues for no residue at all. A pairwise alignment score is the sum of substitution scores and gap penalties over all aligned residues, so that the best alignment is considered to be the one that obtains the highest score [25]. In the case of nucleic acid sequence comparison, scores can be represented in the form of a $4 \times 4$ unitary matrix consisting of ones (or another positive score) on the diagonal for scoring A-A, C-C, G-G and T-T matches and zeros (or a negative score) for scoring mismatches. Although a unitary matrix suffices for typical nucleic acid alignment tasks, alignments of protein sequences benefit from scores that take into account residue-specific information. A 20×20 unitary matrix is outperformed in alignment tasks by matrices that customize scores for each particular amino acid pair, 210 in all (20 possible match scores on the diagonal and 190 possible mismatch scores). As alignment uncertainty increases, the choice of substitution scores (and gap penalties) becomes increasingly important [25]. Because the matrix is symmetrical, only half of the off-diagonal scores are needed to provide a complete scoring scheme for residue substitutions. There are some important matrices in sequence alignment, such as PAM matrix [26], BLOSUM matrix [27] and H3P2 matrix [28]. During the past year, important progress has been made in determining which of these matrices work well for alignment applications that require gap penalties. Clustered Scoring Matrix (e.g. blosum 90 and blosum62), H3P2 matrix, PAM 250 matrix, and GONNET matrices were compared for their ability to detect proteins belonging to the same fold family as the probe but to different sequence families [29]. In a general way, alignment $P$ values drop into the scope, viz. $0 \leq P \leq 1$ Moreover, the more the $P$ value is, the higher the activity is, which reflect the more credible the prediction. Here, we adopt matrix comparison method to analyze DMPRs based on the Chinese herbal medicines, namely Chinese medicinal herbs informatics. Our research results reveal that DMPRs show diversity, complexity, and non-linearity. And our results possess potential application and extensive biological significance.

Usually, $P$ values remain with the scope $(0 \leq P \leq 1)$. Here, the more the $P$ values, the more credible the prediction results, the higher the biological activity. And with the difference of research objects, the scope of $P$ value differs, which is because the limitation of members in training set leads to the difference of prediction veracity. For protein, the sorts of amino acid in training set are less than 20 (e.g. the $20 \times 20$ unitary matrix or special self-constructed matrix aiming at different active sites), and the length of amino acid sequences consisting of training set is often less than the sorts of amino acids occurring in training set, so $P$ values are frequently less than 1 and belong to the extent $(0 \leq P \leq 1)$. The more the $P$ values, the more credible the prediction results; while vice versa. For nucleic acid (RNA or DNA), 4 kinds of nucleotides extremely exist in training set, and the length of nucleotide sequences consisting of training set is often more than 4, so $P$ values are commonly less than 1 and belong to the extent $0 \leq P \leq 1$. The more the $P$ values, the more credible the prediction results; while vice versa. For DMPRs, due to the unilateralism of CMMs in training set whereas the variety of CMMs occurring in DMPRs, the sorts of the medicines consisting of training set are far less than those of the medicines occurring in DMPRs. So, $P$ values are possibly more than 1 or equal to zero, but mostly belong to the extent $(0 \leq P \leq 1)$. However, the size of $P$ value is not in direct ratio to the prediction result but possesses an unambiguous guide. Moreover, the score matrix of CMMs is different from those of protein and nucleic acid. The former scores based on the species of CMMs but not on the sequence, the latter scores not only based on the kinds of the residues but also on the residue orders. And the traditional Chinese medicine demonstrated a good practice and shows a bright future in the therapy of diabetes and its complications because distinctive traditional medical theory emphasizes on integrated care of body and removed symptoms. Compound recipes are often used for diabetes treatment based on the fact that every CMM in compound recipes can provide its special function, and lastly, form an integrated function for treatment of diabetes 
and complications. Single CMM contains multi-ingredients, but these ingredients cannot play such a role as CMMs in compound recipes. Because DMPRs are composed of various CMMs and the effects are related to CMM compositions of the prescriptions but not to the sequences, the score matrix of CMMs take into account whether the species of Chinese herbal medicines in some DMPRs match with those of the training set. This is the most difference from the score matrices of protein and nucleic acid and is also the reason that the $P$ value of DMPRs is not in direct ratio to the prediction result but plays a definite guiding role.

Two training sets of the simple matrix method and the complex matrix method are separately constituted by 8 kinds of DMPRs (Table 2), and severally obtain a $1 \times 18$ unitary matrix of the simple score matrix and a $1 \times 10$ unitary matrix of the first score matrix of the complex matrix. And these CMMs consisting of the matrix possess a percentage of more than $12 \%$, especially membranous milkvetch root (65.66\%) and rehmannia dried rhizome (40.34\%) with a high proportion. To improve prediction of the score matrix and enhance its reliability, take some key mated herbs of treating diabetes to introduce the complex matrix, and construct a $1 \times 12$ unitary matrix of the secondary score matrix (Table 3 ) based on the first matrix and the corresponding CMMs of drug pairs, which possess a percentage of more than $6.01 \%$. In comparison with the simple matrix, the complex matrix possesses higher dependability; the former contains a score matrix but the latter includes two score matrixs: the first matrix and the secondary matrix. Besides 15 same CMMs, namely membranous milkvetch root, common yam rhizome, figwort root, danshen root, Chinese atractylodes rhizome, lobed kudzurine root, asiatic cornelian cherry fruit, rehmannia dried rhizome, radix rehmanniae preparata, white peony root, largehead atractylodes rhizome, mongolian snakegourd root, oriental waterplantain rhizome, Coptic root, and lycium barbarum fruit, the former also includes 3 kinds of CMMs (tuckahoe dried sclerotium, tree peony bark, and Chinese angelica) while the latter contains another 7 kind of CMMs (dwarf lilyturf tuber, red paeony root, common anemarrhena rhizom, orange magnoliavine fruit, Chinese thorowax root, manyflower solomonseal rhizome, and fllase asiabeli root). Moreover, these same herbs have different scores in different matrix due to training set's recipes different.

\section{Conclusion}

Diabetes mellitus is chronic metabolic syndrome and systemic underway disease with high blood glucose, and the third disease in the world just inferior to cancer and cardio-cerebrovascular disease. Cardiovascular disease is the major cause of the morbidity and mortality associated with diabetes in the US. Metabolic syndrome is a disorder comprising of adiposity, dyslipidemia, abnormal glucose tolerance, and hypertension. Inflammation plays a pivotal role in atherosclerosis and is involved in abnormalities associated with metabolic syndrome such as insulin resistance and adiposity. And the traditional Chinese medicine demonstrated a good practice and shows a bright future in the therapy of diabetes and its complications because distinctive traditional medical theory plays emphasis on integrated care of body, and then remove symptoms, which are more effective not only to treat and prevent diabetic complications but also at the meantime to lower blood glucose level. Currently, bioinformatics has already impenetrate biology, medicine, genomics, clinical pharmacology, botany, and other subjects. With the trend of "back-to-the-nature" and the fact of China as a member of the World Trade Organization (WTO), the use, development, and modernization in every aspect of Chinese herbal medicine to cope with the fast growing demand in quality medicines might be boosted and already has brought more challenges to herbal company in China and the world. Thus some attempts for future research and development for CMMs are proposed, especially the application of Chinese traditional medicine informatics to DMPRs. Moreover, these same herbs have different scores in different matrix due to training set's recipes different. Our research results reveal that there is not linearity relationship between $P$ value and clinical activities of DMPRs that show diversity and complexity, which is because the limitation of Chinese medicine compositions in training set influences veracity of prediction. The t-test results reveal that the difference between averages ( $P$ values) of both the simple matrix method and the complex matrix method displays a remarkable significance. And the latter is more logical and more credible than the former because the secondary score matrix of the former plays a complementary role to the first score matrix so that enhance the reliability and the veracity of prediction. In comparison with the simple matrix, the complex matrix possesses higher dependability; the former contains a score matrix but the latter includes two score matrixs: the first matrix and the secondary matrix. Besides 15 same CMMs, the former also includes 3 kinds of CMMs while the latter contains another 7 kind of CMMs. And the size of $P$ value is not in direct ratio to the prediction result but plays a distinct guiding role.

Therefore, considering the complicacy, multiformity, and nonlinearity of Chinese traditional prescriptions, we should ulteriorly develop multi-scale and multi-level analysis on the basis of concrete experimental data. On the one hand, systemically analyze and compare the extracts, herbal medicines, drug paris, and compound recipes of Chinese medicinal materials using grading extraction methods; on the other hand, adopt proteomics, transcriptomics, and genomics to differently express various proteins, and analyze and compare the mechanism and the metabolism pathway of some CMMs in order to identify and validate new potential targets to diabetes. On the contrary, utilize affinity of chromatography technology to separate and purify the available compositions binding with the potential diabetes target, analyze and identify the structures and characters of the available compositions, 
and estimate and appraise their security by metabolomics. Finally, take the available compositions as lead compound, design and synthesize a series of derivants by computeraided drug design, further search and find higher effect and lower toxicity potential candidates in order to find innovative drug. This will greatly change the actualities of CMM research, enhance the probability of bioinformatics applying to CMM research, increase the research level of Chinese traditional medicine informatics, and accelerate the investigation process of Chinese medicinal herbs modernization.

\section{Acknowledgements}

This work was supported by a grant from Basic Scientific Research Expenses of Central University (020814360012) and a grant from National Key Technology R\&D Program (2008BAI51B01).

\section{Competing interests}

The authors declare that they have no competing interests.

\section{Publication history}

Editor: John M. Flack, Wayne State University, USA.

Received: 05-Apr-2012 Revised: 06-June-2012

Accepted: 28-Aug-2012 Published: 19-Sep-2012

\section{References}

1. Musselman DL, Betan E, Larsen H, Phillips LS: Relationship of depression to diabetes types 1 and 2: epidemiology, biology, and treatment. Biol Psychiatry 2003; 54;(3.);317-29. | Article | PubMed

2. Zinman B: Glucose control in type 1 diabetes: from conventional to intensive therapy. Clin Cornerstone 1998; 1;(3.);29-38. | PubMed

3. Gloyn AL: The search for type $\mathbf{2}$ diabetes genes. Ageing Res Rev 2003; 2;(2.);111-27. | Article | PubMed

4. R. Oiknine, A.D. Mooradian, Drug therapy of diabetes in the elderly, Biomed. Pharmacotherapy 57 (2003) 231-239. | Article

5. C. Handschin, V.K. Mootha, Estrogen-related receptor a (ERRa): A novel target in type 2 diabetes. Drug Discovery Today, Therapeutic Strategies 2(2) (2005) 151-156.

6. Li WL, Zheng HC, Bukuru J, De Kimpe N: Natural medicines used in the traditional Chinese medical system for therapy of diabetes mellitus. J Ethnopharmacol 2004; 92;(1.);1-21. | Article | PubMed

7. Chen F, Florkowski CM, Dever M, Beaven DW: Death Certification and New Zealand Health Information Service (NZHIS) statistics for diabetes mellitus: an under-recognised health problem. Diabetes Res Clin Pract 2004; 63;(2.);113-8. | Article | PubMed

8. Chan K: Chinese medicinal materials and their interface with Western medical concepts. J Ethnopharmacol 2005; 96;(1-2.);1-18. | Article $\mid$ PubMed

9. Ou B, Huang D, Hampsch-Woodill M, Flanagan JA: When east meets west: the relationship between yin-yang and antioxidation-oxidation. FASEB J 2003; 17;(2.);127-9. | Article | PubMed

10. Luo Q, Cai Y, Yan J, Sun M, Corke H: Hypoglycemic and hypolipidemic effects and antioxidant activity of fruit extracts from Lycium barbarum. Life Sci 2004; 76;(2.);137-49. | Article | PubMed

11. Tian WX, Li LC, Wu XD, Chen CC: Weight reduction by Chinese medicinal herbs may be related to inhibition of fatty acid synthase. Life Sci 2004; 74;(19.);2389-99. | Article | PubMed

12. Molidor R, Sturn A, Maurer M, Trajanoski Z: New trends in bioinfor- matics: from genome sequence to personalized medicine. Exp Gerontol 2003; 38;(10.);1031-6. | Article | PubMed

13. P.A. Whittaker: The role of bioinformatics in target validation. Drug Discovery Today Technologies 1(2) 2004; 125-133. | Article

14. White CN, Chan DW, Zhang Z: Bioinformatics strategies for proteomic profiling. Clin Biochem 2004; 37;(7.);636-41. | Article | PubMed

15. Philippi S, Kohler J: Using XML technology for the ontology-based semantic integration of life science databases. IEEE Trans Inf Technol Biomed 2004; 8;(2.);154-60. | PubMed

16. Y.-H. Wu, ed., Diabetes Chinese traditional prescriptions. GuangDong Science and technology publishing company, Guangzgou, China, 2002.

17. Yang J, Dong XC, Leng Y: Conformation biases of amino acids based on tripeptide microenvironment from PDB database. J Theor Biol 2006; 240;(3.);374-84. | Article | PubMed

18. Zhan C, Yang J, Dong XC, Wang YL: Molecular modeling of purinergic receptor P2Y12 and interaction with its antagonists. J Mol Graph Model 2007; 26;(1.);20-31. | Article | PubMed

19. Markstein M, Markstein P, Markstein V, Levine MS: Genome-wide analysis of clustered Dorsal binding sites identifies putative target genes in the Drosophila embryo. Proc Natl Acad Sci U S A 2002; 99;(2.);763-8. | Article | PubMed Abstract | PubMed Full Text

20. Chung $\mathrm{SH}$, Choi CG, Park SH: Comparisons between white ginseng radix and rootlet for antidiabetic activity and mechanism in KKAy mice. Arch Pharm Res 2001; 24;(3.);214-8. | PubMed

21. Attele AS, Zhou YP, Xie JT, Wu JA, Zhang L, Dey L, et al:: Antidiabetic effects of Panax ginseng berry extract and the identification of an effective component. Diabetes 2002; 51;(6.);1851-8. | Article | PubMed

22. H.Q. Cheng, Y. Zhang, Y.Y. Guo, The mechanisms of Chinese traditional medicines to lower blood glucose, Xinjiang J. Chinese Traditional Medicine 16 1998; 53-55.

23. H.Y. Ye, M.H. Yu, L. You, Y.Q. Yan, X.F. Yang, Effects of Astragalus polysaccharide on metabolism and heart function of rats with streptozocin-induced diabetes, J. Shanghai Medical University 2000; (27); 357-358.

24. Y.J. Xu, Q.Y. Zhang, Q.W. Wu, Effect of Astragalus membranaceus on experimental diabetic renal hypertrophy and microalbuminuria. Journal of the Shanghai Second Medical University; 1997; (17); 357-359. Article

25. Henikoff S: Scores for sequence searches and alignments. Curr Opin Struct Biol 1996; 6;(3.);353-60. | Article | PubMed

26. M.O. Dayhoff, R.M. Schwartz, B.C. Orcutt, A model of evolutionary change in proteins. Atlas of protein sequence and structure; 1978; (5); 345-352.

27. Henikoff S, Henikoff JG: Amino acid substitution matrices from protein blocks. Proc Natl Acad Sci U S A 1992; 89;(22.);10915-9. | Article | PubMed Abstract $\mid$ PubMed Full Text

28. Rice DW, Eisenberg D: A 3D-1D substitution matrix for protein fold recognition that includes predicted secondary structure of the sequence. J Mol Biol 1997; 267;(4.);1026-38. | Article | PubMed

29. Bastien O, Roy S, Marechal E: Construction of non-symmetric substitution matrices derived from proteomes with biased amino acid distributions. CR Biol 2005; 328;(5.);445-53. | PubMed 